\title{
Reconstruction of sub cross-correlation cancellation technique for unambiguous acquisition of $\operatorname{BOC}(k n, n)$ signals
}

\author{
JI Yuanfa ${ }^{1,2}$, CHEN Xiaoqian ${ }^{1,2}$, FU Qiang ${ }^{1,2}$, SUN Xiyan ${ }^{1,2, *}$, and ZHEN Weimin ${ }^{3}$ \\ 1. Guangxi Key Laboratory of Precision Navigation Technology and Application, Guilin University of Electronic Technology, \\ Guilin 541004, China; 2. National \& Local Joint Engineering Research Center of Satellite Navigation Positioning and Location \\ Service, Guilin 541004, China; 3. Key Laboratory of Radio Wave Environment and Modeling Technology, Qingdao 266000, China
}

\begin{abstract}
In order to solve the problem of ambiguous acquisition of BOC signals caused by its property of multiple peaks, an unambiguous acquisition algorithm named reconstruction of sub cross-correlation cancellation technique (RSCCT) for $\operatorname{BOC}(k n, n)$ signals is proposed. In this paper, the principle of signal decomposition is combined with the traditional acquisition algorithm structure, and then based on the method of reconstructing the correlation function. The method firstly gets the sub-pseudorandom noise (PRN) code by decomposing the local PRN code, then uses $\operatorname{BOC}(k n, n)$ and the sub-PRN code cross-correlation to get the sub cross-correlation function. Finally, the correlation peak with a single peak is obtained by reconstructing the sub cross-correlation function so that the ambiguities of $\mathrm{BOC}$ acquisition are removed. The simulation shows that RSCCT can completely eliminate the side peaks of $\mathrm{BOC}(k n, n)$ group signals while maintaining the narrow correlation of $\mathrm{BOC}$, and its computational complexity is equivalent to sub carrier phase cancellation (SCPC) and autocorrelation side-peak cancellation technique (ASPeCT), and it reduces the computational complexity relative to $\operatorname{BPSK}$-like. For $\operatorname{BOC}(n, n)$, the acquisition sensitivity of RSCCT is $3.25 \mathrm{~dB}, 0.81 \mathrm{~dB}$ and $0.25 \mathrm{~dB}$ higher than binary phase shift keying (BPSK)-like, SCPC and $\mathrm{ASPeCT}$ at the acquisition probability of $90 \%$, respectively. The peak to average power ratio is 1.91, 3.0 and 3.7 times higher than ASPeCT, SCPC and BPSK-like at SNR $=-20 \mathrm{~dB}$, respectively. For $\operatorname{BOC}(2 n, n)$, the acquisition sensitivity of RSCCT is $5.5 \mathrm{~dB}, 1.25 \mathrm{~dB}$ and $2.69 \mathrm{~dB}$ higher than BPSK-like, SCPC and ASPeCT at the acquisition probability of $90 \%$, respectively. The peak to average power ratio is $1.02,1.68$ and 2.12 times higher than ASPeCT, SCPC and BPSK-like at SNR $=-20 \mathrm{~dB}$, respectively.
\end{abstract}

Keywords: signal decomposition, sub cross-correlation, reconstructing correlation function, acquisition performance.

DOI: $10.21629 /$ JSEE.2019.05.04

\footnotetext{
Manuscript received March 06, 2018

*Corresponding author.

This work was supported by the National Science Foundation of China (61561016; 61861008; 11603041), the Guangxi Natural Science Foundation Project (2018JJA170090), the Innovation Project of Guet Graduate Education (2018YJCX19; 2018YJCX31), and Guangxi Key Laboratory of Precision Navigation Technology and Application, Guilin University of Electronic Technology (DH201707).
}

\section{Introduction}

With the development of radio technology and the increasing demand of people, the global satellite navigation technology is booming. Crowded frequency band resources, positioning accuracy and reliability have become an urgent problem to be solved. With the birth and development of major global navigation systems as well as military and civilian applications in different aspects, navigation signals of different modulation modes need to share the same frequency band. With the development of urban architecture, it is required that the navigation signal has some anti-multipath effect to ensure positioning accuracy. Considering the requirements in the above two aspects of the user, the binary offset carrier (BOC) modulation was introduced [1]. The signal energy of the BOC signal is mainly distributed on both sides of the center frequency so that it can be well compatible with the binary phase shift keying (BPSK) signal [2]. And its auto-correlation peak is steeper than BPSK signal's, which has better antimultipath performance and higher tracking accuracy. However, the multi-peak property of the BOC correlation function results in ambiguity in both its acquisition and tracking, which may be mistakenly synchronized to the sidepeak of the correlation function, so it is critical to master the unambiguous synchronization technique of BOC.

With the introduction of the BOC modulation, many acquisition methods for the BOC signal are proposed, which are mainly divided into the ones based on the traditional BPSK spectrum and reconstruction of the correlation function. The typical processing algorithms based on the traditional BPSK spectrum are as follows: The traditional parallel code phase search algorithm is applied to the BOC signal, which can be successfully caught under the condition of high signal-to-noise ratio (SNR), but basically belongs to the ambiguous acquisition algorithm [3]. The 
BPSK-like algorithm takes advantage of the characteristics of BOC, which is similar to moving the BPSK signal to two sides of the spectrum; the local pseudorandom noise (PRN) code is moved correspondingly; then BOC is captured by the method suitable for BPSK. Although BPSKlike can remove the ambiguities of BOC and its structure is simple, its correlation peak similar to BPSK and there is no advantage of the narrow correlation [4]. Sub carrier phase cancellation (SCPC) uses a pair of orthogonal local BOC codes to remove the effect of sub-carrier on the acquisition of the BOC signal, and the result is similar to BPSK-like [5].

This kind of acquisition algorithm based on traditional BPSK removes ambiguities by sacrificing the narrow correlation of BOC. Consequently, this kind of algorithm has not been widely used. And, the processing method based on reconstruction of the correlation function has become a research hot-spot, in which autocorrelation side-peak cancellation technique (ASPeCT) has attracted intense attention [6]. The essence of ASPeCT is that the received signal wipping off the carrier is not only correlated with the local BOC code but also correlated with the local PRN code, and then obtains a new correlation function by these two correlation values to eliminate ambiguities. This method can effectively restrain the side peak of the BOC correlation and maintain the narrow correlation, but it is only suitable for $\operatorname{BOC}(n, n)$. The acquisition algorithm based on fractal reconstruction can get the correlation function without side peaks by folding [7]. Its computation complexity is low; the correlation peak is $43.7 \%$ higher than that of the $\mathrm{ASPeCT}$ algorithm; and it is suitable for $\operatorname{BOC}(k n, n)$ signals. However, this method makes the peak slope steeper by increasing the amplitude of the main peak, so the main peak width is similar to that of BPSK. Ji et al. [8] proposed an acquisition algorithm that is significantly superior to ASPeCT in terms of computation and sensitivity. The algorithm divides the local BOC code into odd and even units, and then reconstructs the correlation function between the parity unit and the received signal, but the algorithm is also only suitable for $\operatorname{BOC}(n, n)$. In this paper, an unambiguous acquisition algorithm belonging to this class is presented. It is based on the combination of two sub cross-correlation functions that remove the inherent $\mathrm{BOC}(k n, n)$ auto-correlation side peaks.

This paper is organized as follows: Section 2 gives the principle of reconstruction of sub cross-correlation cancellation technique (RSCCT); Section 3 describes the the analysis process of detection performance; performance evaluation results obtained by theoretical analysis and simulations are provided in Section 4; finally, some conclusions are drawn in the last section.

\section{Principle of RSCCT}

\subsection{Correlation characteristics of $\operatorname{BOC}(k n, n)$}

$\mathrm{BOC}(k n, n)$ modulation signals are derived from traditional GNSS signals [9], and only multiply a squarewave sub-carrier on the basis of the BPSK modulation. $\mathrm{BOC}(k n, n)$ signals in time domain can be modeled as

$$
s_{\mathrm{BOC}(k n, n)}(t)=A d(t) c(t) \operatorname{sign}\left(\sin \left(2 \pi f_{s c} t\right)\right)
$$

where $A$ is the signal's amplitude; $d(t)$ represents the navigation data; $c(t)$ is the PRN spreading code; and $\operatorname{sign}\left(\sin \left(2 \pi f_{\text {sub }} t\right)\right)$ represents the square-wave sub-carrier. Using $f_{0}=1.023 \mathrm{MHz}$ as the reference frequency, $\operatorname{BOC}(k n, n)$ is characterized by its spreading code $f_{c}=$ $n f_{0}$, and its sub-carrier frequency $f_{s c}=k n f_{0} . N=$ $2 k n / n=2 k$ is defined as the modulation order of $\operatorname{BOC}(k n, n)$. The product of PRN and sub-carrier is defined as the BOC code, which is expressed as

$$
s_{\mathrm{BOC}}(t)=c(t) \operatorname{sign}\left(\sin \left(2 \pi f_{s c} t\right)\right) .
$$

Because of the correlation characteristics of the two parts of the PRN code and the sub-carrier, $\operatorname{BOC}(k n, n)$ has multiple correlation peaks. The multi-peak feature of the correlation function will not only cause ambiguities of acquisition, but also need specific algorithms for capturing BOC signals with different orders. The auto-correlation function $R_{B}(\tau)$ of $\operatorname{BOC}(k n, n)$ and its cross-correlation function $R_{B / P}(\tau)$ with PRN can be given $[10,11]$ by

$$
\begin{gathered}
R_{B}(\tau)=\sum_{i=1}^{N-1}\left\{\left[(-1)^{i} t r i_{-\frac{i}{N}}(\tau)+(-1)^{i} \operatorname{tri} \frac{i}{N}(\tau)\right] .\right. \\
\left.\frac{N-i}{N}\right\}+t r i_{0}(\tau) \\
R_{B / P}(\tau)= \\
\sum_{i=1}^{N / 2}\left\{\left[\operatorname{tri}-\frac{2 i-1}{N}(\tau)+(-1) \operatorname{tr} i_{\frac{2 i-1}{N}}(\tau)\right] \cdot \frac{1}{N}\right\}
\end{gathered}
$$

where $\tau$ is the code phase delay; $N$ is the modulation order of BOC; $t r i_{\frac{i}{N}}$ represents the triangular function of peak at $\frac{i \times T c}{N}$; and $T_{c}=1 / f_{c}$ represents a PRN code period. Fig. 1 shows the comparison of the auto-correlation functions of $\operatorname{BPSK}, \operatorname{BOC}(n, n)$ and $\operatorname{BOC}(2 n, n)$ signals, and Fig. 2 is the comparison of cross-correlation functions. 


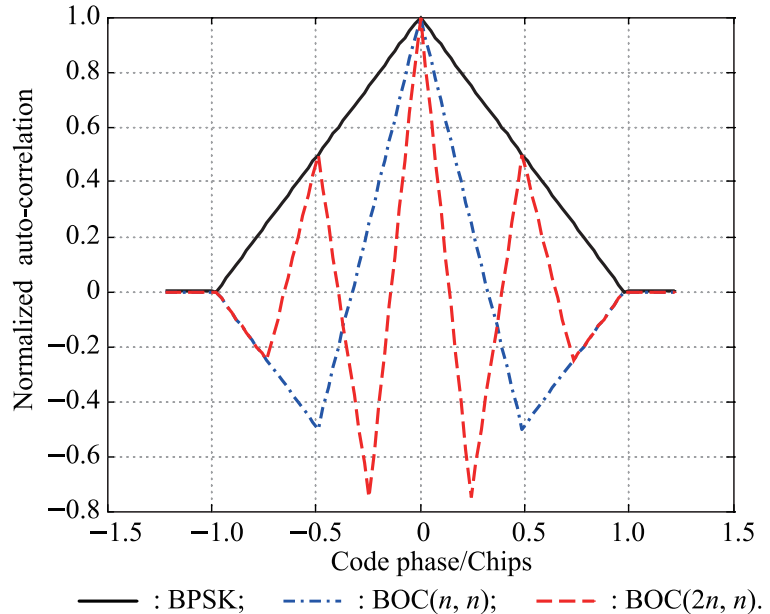

Fig. 1 Auto-correlation function

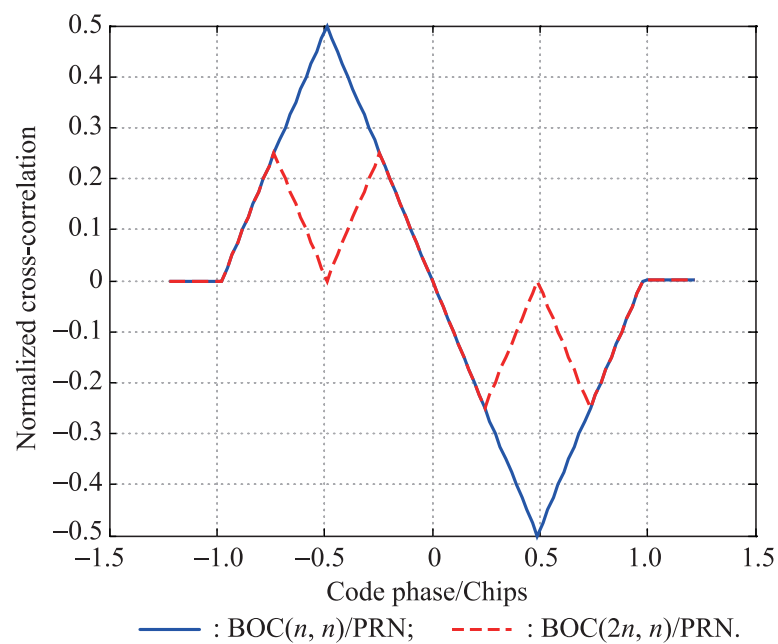

Fig. 2 Cross-correlation function

\subsection{Generation of sub-PRN code sequences}

Equation (2) with the form of rectangular pulse can be modeled as

$$
s_{\mathrm{BOC}}(t)=\sum_{j=-\infty}^{+\infty} \sum_{i=1}^{N}\left[c(t)(-1)^{i-1} p_{i}\left(t-j T_{c}\right)\right]
$$

where $p_{i}(t)$ represents a pulse signal and its expression is

$$
p_{i}(t)=\left\{\begin{array}{ll}
1, & (i-1) T_{c} / N \leqslant t \leqslant i T_{c} / N \\
0, & \text { otherwise }
\end{array} .\right.
$$

Similarly, the PRN code can be expressed by

$$
s_{c}(t)=\sum_{i=1}^{N}\left[c(t) p_{i}(t)\right]=\sum_{i=1}^{N} s_{c, i}(t)
$$

where $s_{c, i}(t)$ is defined as the $i$ th sub-PRN code, which means that each PRN code chip is divided into $N$ segments; only the $i$ th segment of the PRN is retained; and the others are set to zero. Fig. 3 and Fig. 4 respectively show the sub codes of PRN code decomposed at $N=2,4$.
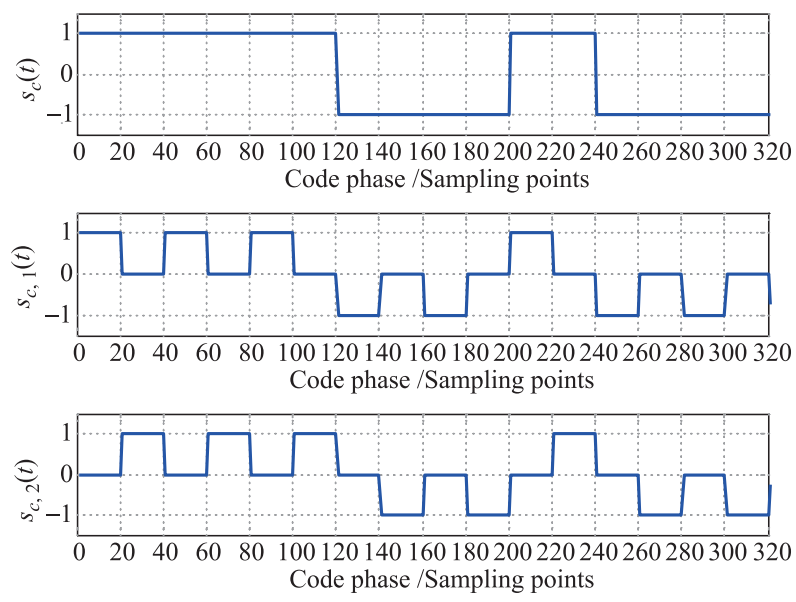

Fig. 3 Decomposition of PRN at $N=2$
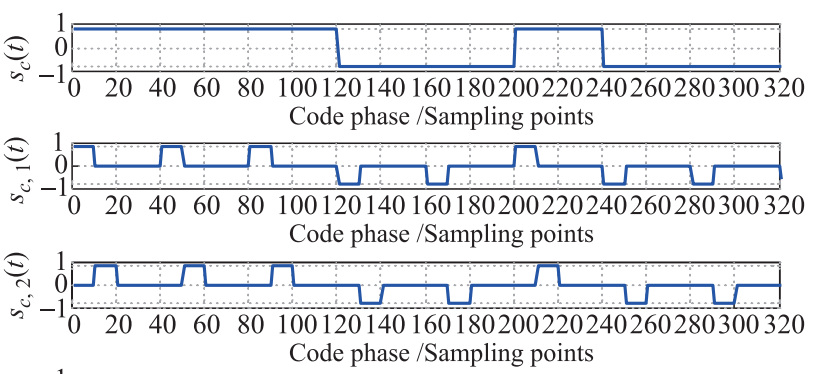

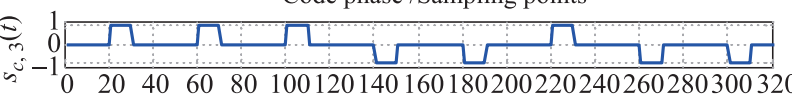

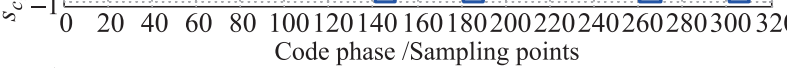

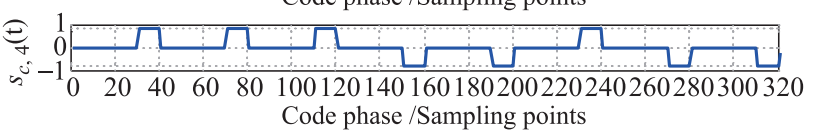

Fig. 4 Decomposition of PRN at $N=4$

\subsection{Generation of sub-PRN code sequences}

It is assumed that the cross-correlation characteristics between $\mathrm{BOC}(k n, n)$ and PRN sequences are ideal, and the coherent integration time is $T_{\text {coh }}$, then the cross-correlation function $R_{B / P}(\tau)$ of the BOC code and PRN code is

$$
\begin{gathered}
R_{B / P}(\tau)=\frac{1}{T_{\mathrm{coh}}} \int_{0}^{T_{\mathrm{coh}}} s_{\mathrm{BOC}}(t) \cdot s_{c}(t+\tau) \mathrm{d} t= \\
\frac{1}{T_{\mathrm{coh}}} \frac{T_{\mathrm{coh}}}{T_{c}} \int_{-\tau}^{T_{c}} s_{\mathrm{BOC}}(t) \cdot s_{c}(t+\tau) \mathrm{d} t= \\
\sum_{i=1}^{N} R_{B / P i}(\tau)
\end{gathered}
$$

where

$$
R_{B / P i}(\tau)=\frac{1}{T_{c}} \int_{0}^{T_{c}} \sum_{j=1}^{N}(-1)^{j-1} p_{j}(t) p_{i}(t+\tau) \mathrm{d} t .
$$


According to (5) to (9), it can be further expressed as

$$
R_{B / P i}(\tau)=\frac{1}{T_{\mathrm{coh}}} \int_{0}^{T_{\mathrm{coh}}} s_{\mathrm{BOC}}(t) \cdot s_{c, i}(t+\tau) \mathrm{d} t .
$$

Therefore, $R_{B / P i}(\tau)$ can be defined as the sub crosscorrelation function of the BOC code and the $i$ th sub-PRN code. Fig. 5 and Fig. 6 respectively show the normalized sub cross-correlation function at $N=2,4$.
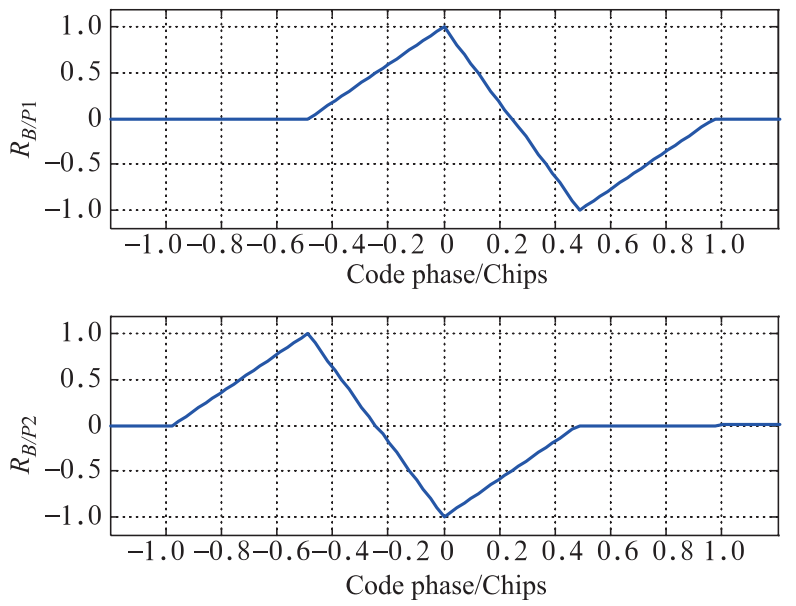

Fig. 5 Normalized sub cross-correlation functions at $N=2$

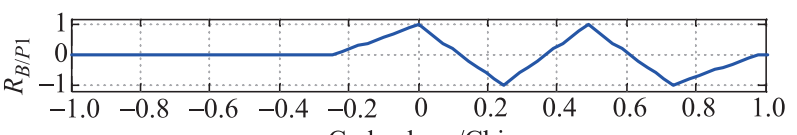
Code phase/Chips
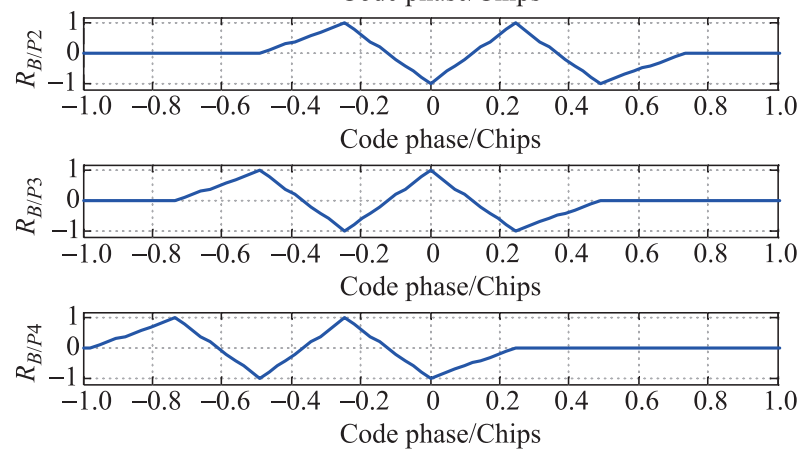

Fig. 6 Normalized sub cross-correlation functions at $N=4$

It can be seen from Fig. 5 and Fig. 6, no matter what value $N$ is, the product of $R_{B / P 1}(\tau)$ and $R_{B / P N}(\tau)$ can yield non-zero value only near the $(0,0)$ point. In addition, if $R_{B / P 1}(\tau)$ rotates $180^{\circ}$ at the $(0,0)$ point, it will get $R_{B / P N}(\tau)$. Therefore, after negating the product of $R_{B / P 1}(\tau)$ and $R_{B / P N}(\tau)$, a correlation function $R_{B / P N}(\tau)$ with one positive main peak and two negative side peaks can be obtained, which is expressed as

$$
R_{M}=-R_{B / P 1} R_{B / P N} .
$$

Considering the detection volume of acquisition output is generally the form of $|\cdot|$ or $|\cdot|^{2}$, in order to further remove the side peaks and increase the value of the main peak, the final reconstruction of the correlation function is equal to

$$
\begin{gathered}
R_{\mathrm{RSC}}=\left|R_{M}+\right| R_{M}||= \\
|| R_{B / P 1} R_{B / P N}\left|-R_{B / P 1} R_{B / P N}\right| .
\end{gathered}
$$

According to the above principles, Fig. 7 shows the normalized $R_{M}$ and $R_{\mathrm{RSC}}$ for $N=2$ and $N=4$. With the increase of the modulation order, the width of the main peak, which is reconstructed by the correlation function, will also be narrowed accordingly. At $N=2$ and $N=4$, the theoretical width of the main peak should be 1 and 0.5 respectively, and $R_{\mathrm{RSC}}$ is smaller than the theoretical value, so it has a better narrow correlation performance.

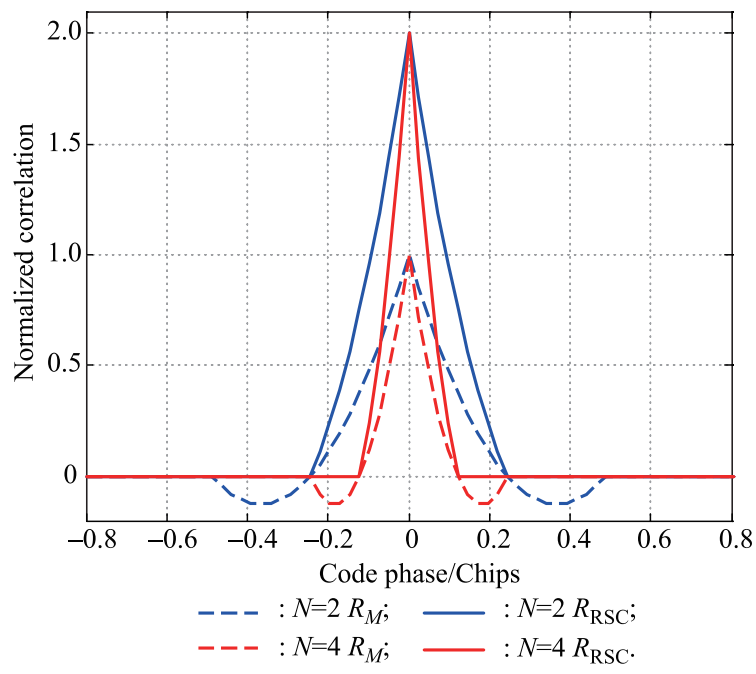

Fig. 7 Reconstruction of correlation functions by RSCCT

\section{Detection performance analysis}

The intermediate frequency signal received by the receiver can be expressed as

$r_{\mathrm{IF}}(t)=\sqrt{2} A d(t-\tau) S_{\mathrm{BOC}}(t-\tau) \cos \left(2 \pi f_{\mathrm{IF}} t+\theta\right)+N_{n}$

where $\sqrt{2} A$ is the signal's amplitude; $d(t)$ represents the navigation data with a level of $\pm 1 ; f_{\mathrm{IF}}$ is the intermediate frequency; $\theta$ is the initial phase of carrier; $\tau$ is the code phase delay; and $N_{n}$ is assumed to be additive Gaussian white noise with a mean of zero and a variance of $\sigma^{2}$ [12]. The received signal after the carrier is wiped off is equal to

$$
r(t)=\sqrt{2} A d(t) S_{\mathrm{BOC}} \cdot \mathrm{e}^{\mathrm{j}(2 \pi \Delta f t+\theta)}+N_{n}
$$

where $\Delta f$ is the estimation error for the carrier frequency.

Supposing the correlation solution of the received signal and the local PRN code is $R(\tau)$, and $d(t)$ will not change in $T_{\text {coh }}$, then the result of the coherent integral output is expressed as

$r_{p}(t)=A d(t) R(\tau) \sin c\left(\pi \Delta f T_{\text {coh }}\right) \mathrm{e}^{\mathrm{j}\left(2 \pi \Delta f T_{\text {coh }}+\theta\right)}+N_{n} \approx$ 


$$
\operatorname{Ad}(t) R(\tau) \sin c\left(\pi \Delta f T_{\mathrm{coh}}\right)+N_{n}
$$

According to the principle of RSCCT, after generating the local PRN, the first sub-PRN code $s_{c, 1}(t)$ and the $N$ th sub-PRN code $s_{c, N}(t)$ are extracted by time division components, and then multiply with $r(t)$. The result of coherent integration is obtained as follows:

$$
\begin{gathered}
S_{1}=A d(t) R_{B / P 1}(\tau) \sin c\left(\pi \Delta f T_{\mathrm{coh}}\right)+N_{1}= \\
S_{1}(\tau, \Delta f)+N_{1} \\
S_{N}=A d(t) R_{B / P N}(\tau) \sin c\left(\pi \Delta f T_{\mathrm{coh}}\right)+N_{N}= \\
S_{N}(\tau, \Delta f)+N_{N} .
\end{gathered}
$$

Supposing the non-coherent accumulation time is $N_{n c}$, the test statistics is

$$
\begin{gathered}
V_{=} \sum_{m=1}^{N_{n c}}\left[\left|S_{1} S_{N}\right|-\left(S_{1} S_{N}\right)\right] \approx 2 \sum_{m=1}^{N_{n c}}\left[\left|S_{1} S_{N}\right|\right]= \\
2 \sum_{m=1}^{N_{n c}}\left[\left|S_{1}(\tau, \Delta f)+N_{1}\right| \cdot\left|S_{N}(\tau, \Delta f)+N_{N}\right|\right]= \\
2 \sum_{m=1}^{N_{n c}}\left[\left|S_{1}(\tau, \Delta f)\right| \cdot\left|S_{1}(\tau, \Delta f)\right|\right]+ \\
2 \sum_{m=1}^{N_{n c}}\left[\left|S_{1}(\tau, \Delta f)\right| \cdot N_{N}\right]+2 \sum_{m=1}^{N_{n c}}\left[\left|S_{N}(\tau, \Delta f)\right| \cdot N_{1}\right]+ \\
2 \sum_{m=1}^{N_{n c}}\left(N_{1} N_{N}\right) .
\end{gathered}
$$

Equation (18) can be decomposed into

$$
\begin{gathered}
A_{V}=2 \sum_{m=1}^{N_{n c}}\left[\left|S_{1}(\tau, \Delta f)\right| \cdot\left|S_{N}(\tau, \Delta f)\right|\right] \\
H_{V}=2 \sum_{m=1}^{N_{n c}}\left[\left|S_{1}(\tau, \Delta f)\right| \cdot N_{N}\right]+ \\
2 \sum_{m=1}^{N_{n c}}\left[\left|S_{N}(\tau, \Delta f)\right| \cdot N_{1}\right] \\
N_{V}=2 \sum_{m=1}^{N_{n c}}\left(N_{1} N_{N}\right) .
\end{gathered}
$$

It can be understood from (19) to (21) that, $A_{V}$ represents a pure signal with a mean value of $4 N_{n c} A^{2} T_{\text {coh }}^{2}\left|R_{F}(\tau)\right|\left|R_{L}(\tau)\right|$ and a variance of $0 . H_{V}$ denotes the amount of containing signals and noise with mean 0 and variance $2 N_{n c} A^{2}\left(R_{F}(\tau)+R_{L}(\tau)\right)^{2} \sigma^{2}, N_{V}$ is a Gaussian white noise with mean 0 and variance $4 N_{n c} \sigma^{4}$ [13]. Therefore, the mean value $E(V)$ and the variance $D(V)$ for $V$ are as follows:

$$
E(V)=4 N_{n c} A^{2}\left|R_{B / P 1}(\tau)\right|\left|R_{B / P N}(\tau)\right|
$$

$D(V)=4 N_{n c} \sigma^{4}+2 N_{n c} A^{2}\left(R_{B / P 1}(\tau)+R_{B / P N}(\tau)\right)^{2} \sigma^{2}$.

Supposing there are two cases of $H_{0}$ and $H_{1} . H_{0}$ indicates Gauss white noise only. In this condition $V$ is Rayleigh distribution [14], and its mean and variance are respectively expressed as

$$
\begin{aligned}
& E\left(V \mid H_{0}\right)=0 \\
& D\left(V \mid H_{1}\right)=0 .
\end{aligned}
$$

$H_{1}$ indicates that both signals and noise exist. In this condition $V$ obeys Rician distribution, and its mean and variance are expressed as follows:

$$
\begin{gathered}
E\left(V \mid H_{0}\right)=4 N_{n c} A^{2}\left|R_{B / P 1}(\tau)\right|\left|R_{B / P N}(\tau)\right| \\
D\left(V \mid H_{1}\right)=4 N_{n c} \sigma^{4}+2 N_{n c} A^{2}\left(R_{B / P 1}(\tau)+\right. \\
\left.R_{B / P N}(\tau)\right)^{2} \sigma^{2} .
\end{gathered}
$$

It is assumed that the prior probabilities are $P\left(H_{0}\right)$ and $P\left(H_{1}\right)$, and the detection threshold is $V_{t h}$. The false alarm probability $P_{f a}$ and the detection probability $P_{d}$ of $V$ can be obtained as follows:

$$
\begin{gathered}
P_{f a}=\int_{V_{t h}}^{+\infty} P\left(V \mid H_{0}\right) \mathrm{d} V \\
P_{d}=\int_{V_{t h}}^{+\infty} P\left(V \mid H_{1}\right) \mathrm{d} V .
\end{gathered}
$$

\section{Simulation and analysis}

\subsection{Effectiveness of de-ambiguity and generality}

In order to verify the effectiveness of de-ambiguity [15] and generality of RSCCT, the 2-dimensional diagram of the normalized correlation peak with the change of the code phase is shown in Fig. 8 and Fig. 9 when $N=2$ and $N=4$, and using BPSK-like, SCPC, ASPeCT and RSCCT acquisition schemes. The simulation environment is as follows, frequency of sampling is $f_{S}=40.920 \mathrm{MHz}$; Doppler shift is $f_{d}=1500 \mathrm{~Hz}$; code phase is 800 (sampling points); the range of Doppler search is $[-10 \mathrm{KHz}$, $10 \mathrm{KHz}$; and Doppler stepping is $500 \mathrm{~Hz}, \mathrm{SNR}=0 \mathrm{~dB}$.

It can be observed from Fig. 8 and Fig. 9 that although these four algorithms all can remove the ambiguities for $N=2$, the main peak width of BPSK-like and SCPC is $2 T c$ consistent with the BPSK modulation signal, which sacrifices the advantage of the narrow correlation characteristic of BOC modulation signals. ASPeCT has a side peak with an amplitude of 0.1687 when the code phase is $\pm 0.35 T c$, and RSCCT can completely remove the side peak, and the main peak width is only $25 \%$ of the BPSK signal equal to $0.5 T c$. 


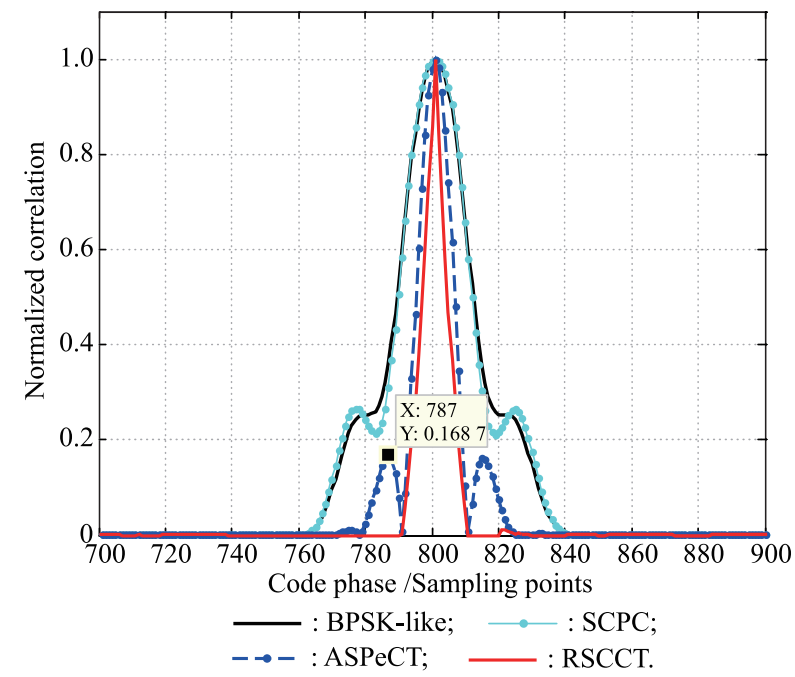

Fig. 8 The 2-D acquisition result at $N=2$

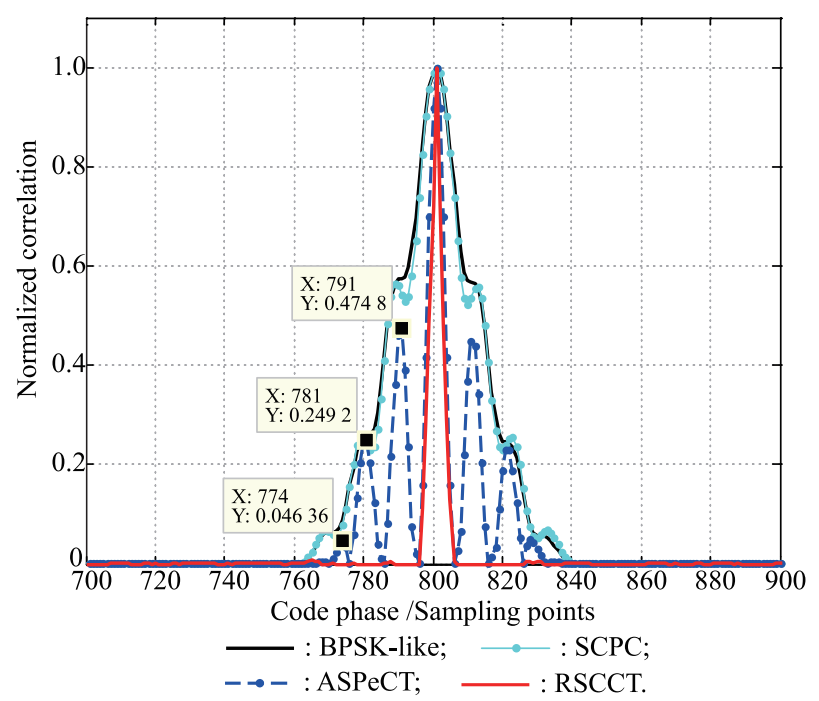

Fig. 9 2-D acquisition result at $N=4$

Therefore, RSCCT can not only effectively remove the ambiguity of signal acquisition of the $\operatorname{BOC}(n, n)$ group, but also preserve the narrow correlation of BOC signals better than the other three algorithms. When $N=4$, BPSK-like and SCPC can effectively remove the related side peaks, but cannot retain the advantage that the correlation main peak of the high-order BOC will be narrowed with the increase of the modulation order, and also degenerate to obtain the same correlation peak width as BPSK. ASPeCT has side peaks of $0.4748,0.2492$ and 0.04636 at $\pm 0.25 T c, \pm 0.5 T c$ and $\pm 0.675 T c$, respectively, which cannot remove the acquisition ambiguity for high-order BOC signals. RSCCT can still completely remove all the side peaks of the 4th-order BOC, and the main peak width is only $0.3 T c$ equal to $60 \%$ of the 2 nd order BOC and $30 \%$ of the BPSK. The algorithm effectively exerts the narrow correlation peak advantage of the high-order BOC signal.

\subsection{Comparison of computation complexity}

The block diagram of RSCCT is illustrated in Fig. 10. The algorithm is simple in structure. Its carrier peeling module is consistent with the traditional one. And in the local code generation module, BPSK-like has to move the PRN code up and down through two multiplications. ASPeCT needs to generate PRN code and BOC code, and RSCCT generates two sub-PRN codes.

Simulate the $1 \mathrm{~ms}$ received signal under the same simulation environment as Section 4.1. The frequency search times are 41 , and the sample point is 40920 , so an FFT is required to operate on 44 points. One complex multiplication is equal to 4 real multiplications and 2 real additions, and one complex plus is equal to 2 real numbers plus. Thus BPSK-like requires 122893072 real multiplications and 104438152 real additions. Both SCPC and ASPeCT requires 122893072 real multiplications and 104438152 real additions. RSCCT requires 122893072 real multiplications and 104438152 real additions. The required real multiplication ratio of BPSK-like, SCPC, ASPeCT and RSCCT is 1.000 0: 0.863 5: 0.863 5: 0.8771 , and the required real addition ratio is 1.000 0: 0.935 7: 0.9357 : 0.935 7. Therefore, RSCCT can save more than $15 \%$ of multiplication and $6.5 \%$ of addition operation compared with the BPSK-like algorithm, which is similar to SCPC and ASPeCT.

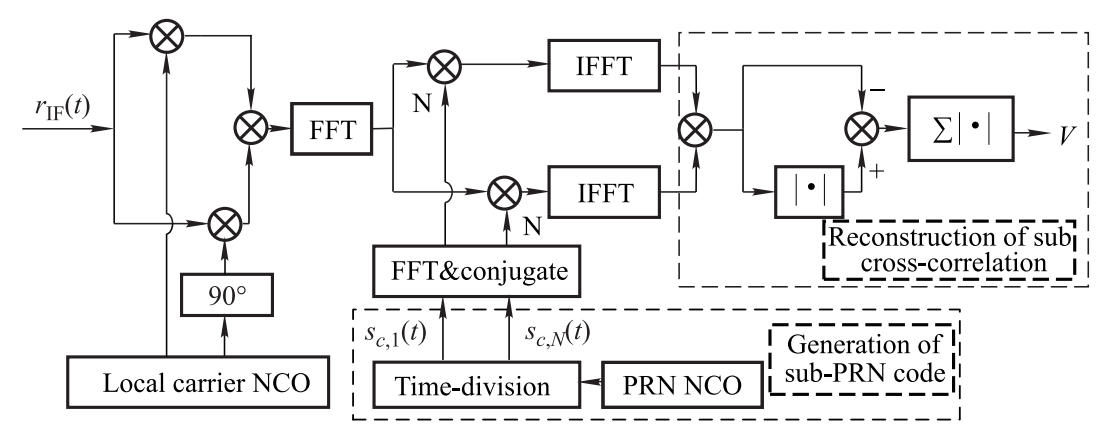

Fig. 10 Block diagram of RSCCT 


\subsection{Acquisition probability}

Set the SRN range of the simulation environment to [-40 -20], and other simulation conditions are consistent with Section 4.1. The comparison of detection probability obtained by repeating 3000 acquisition experiments using BPSK-like, SCPC, ASPeCT and RSCCT algorithms under different SNRs is shown in Fig. 11 and Fig. 12. The basis of correct detection is that the error between the code phase detected by the algorithm and the set code phase is less than $1 / 8 T c$. The ratio of the correct detection times to the total number of experiments is the acquisition probability [16].

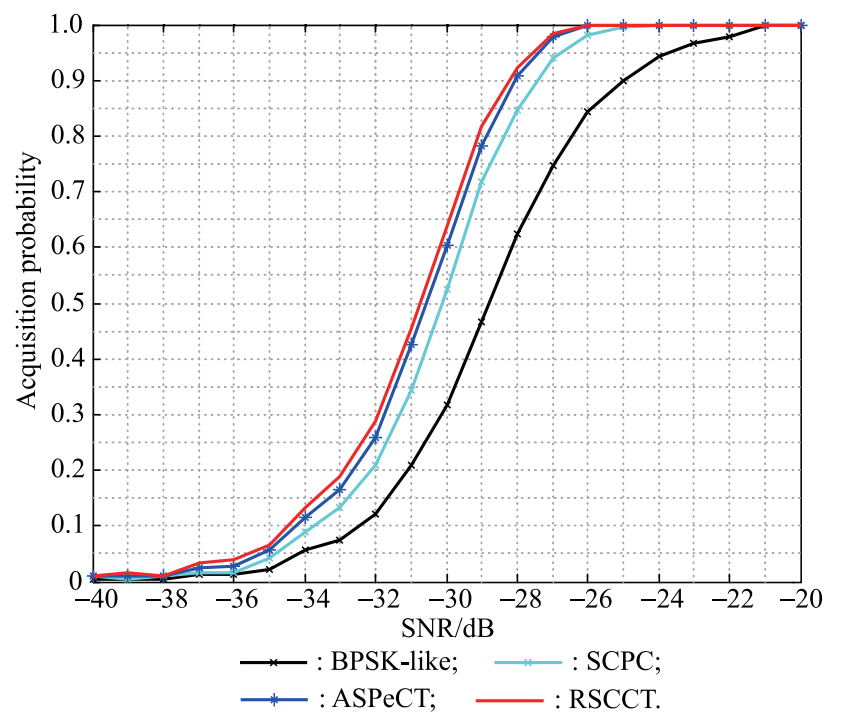

Fig. 11 Acquisition probability at $N=2$

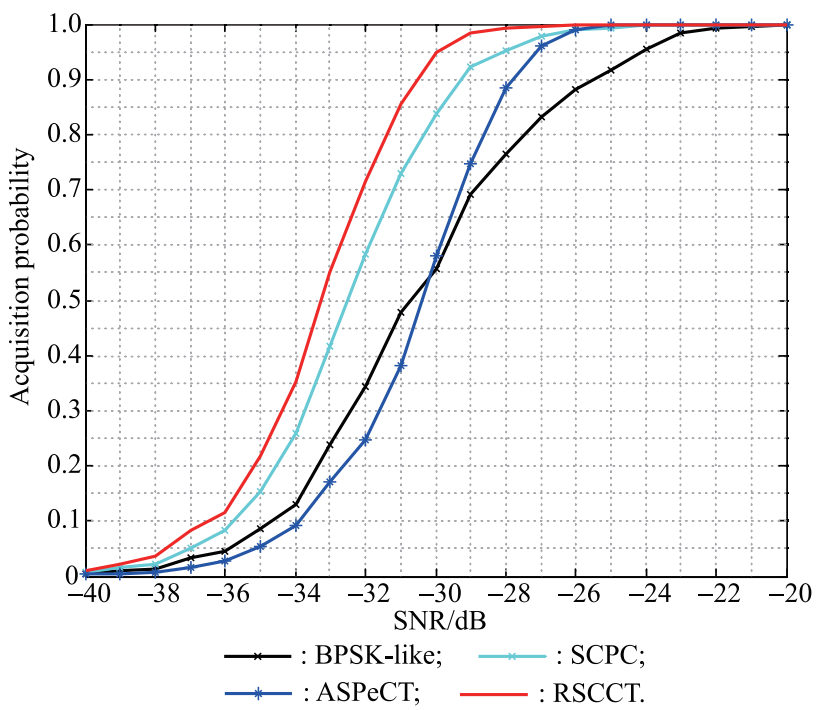

Fig. 12 Acquisition probability at $N=4$

As can be seen from Fig. 11 and Fig. 12, when $N=2$, the acquisition probability of the four methods is ranked as RSCCT, ASPeCT, SCPC and BPSK-like. When the required acquisition probability is $90 \%$, the acquisition sensitivity of RSCCT is $3.25 \mathrm{~dB}, 0.81 \mathrm{~dB}$ and $0.25 \mathrm{~dB}$ higher than BPSK-like, SCPC and ASPeCT respectively. When $N=4$, the acquisition probability of ASPeCT is lower than that of the other three algorithms when the SNR is less than $-30 \mathrm{~dB}$ because it cannot effectively suppress the side peaks, and is less than BPSK-like until the SNR reaches $-30 \mathrm{~dB}$. When the acquisition probability is $90 \%$, the acquisition sensitivity of RSCCT is $5.5 \mathrm{~dB}, 1.25 \mathrm{~dB}$ and $2.69 \mathrm{~dB}$ higher than BPSK-like, SCPC and ASPeCT, respectively. It can be seen that the acquisition probability of the RSCCT algorithm in different BOC modulation orders and different SNRs is higher than that of the BPSKlike and ASPeCT algorithm.

\subsection{Peak to average power ratio}

Peak to average power ratio is also an important indicator for judging the capture performance of different algorithms, and is defined as the ratio of the peak value to the average value of the correlation result obtained by acquisition. In the same simulation environment as Section 4.3, the peak to average power ratio of the four methods under different SNRs are obtained by repeating 3000 experiments as shown in Fig. 13 and Fig. 14.

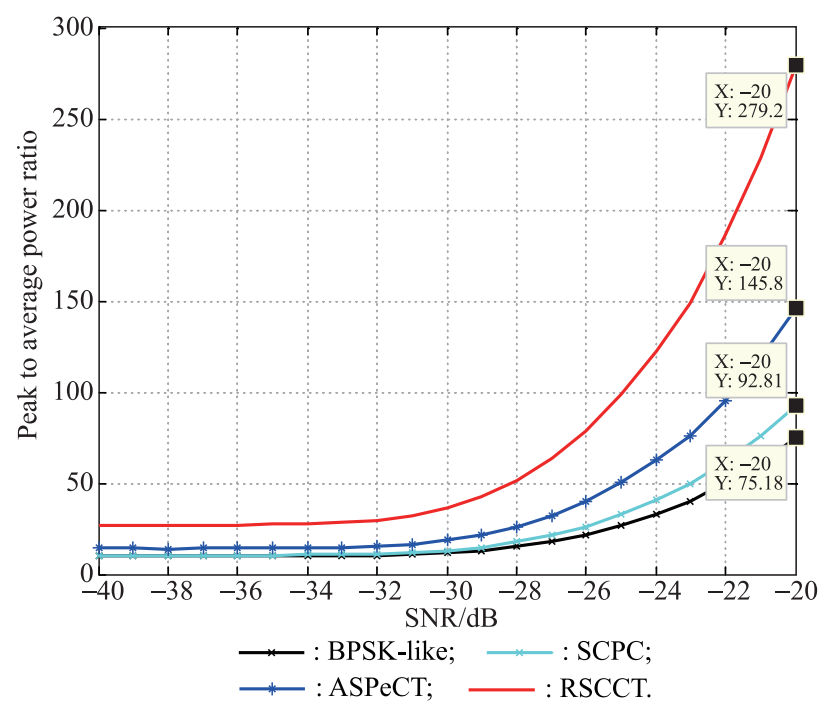

Fig. 13 Peak to average power ratio at $N=2$

It can be seen that RSCCT keeps the highest performance compared with the other three algorithms under different SNRs for $N=2$, and the ranking of the four algorithms is RSCCT, ASPeCT, SCPC and BPSK-like. When SNR $=-20 \mathrm{~dB}$, the value of the four algorithms are 279.2, 145.8, 92.81 and 75.18, respectively. And the results of RSCCT are 1.91, 3.0, and 3.7 times higher than ASPeCT, SCPC, and BPSK-like, respectively. When $N=4$, 
RSCCT remains the highest under different SNRs. ASPeCT is basically the same as RSCCT after the SNR is higher than $-28 \mathrm{~dB}$. When SNR $=-20 \mathrm{~dB}$, the value of the four algorithms are 291.8, 284.7, 173.8 and 137.5 respectively. And the results of RSCCT are 1.02, 1.68 and 2.12 times higher than ASPeCT, SCPC, and BPSK-like, respectively.

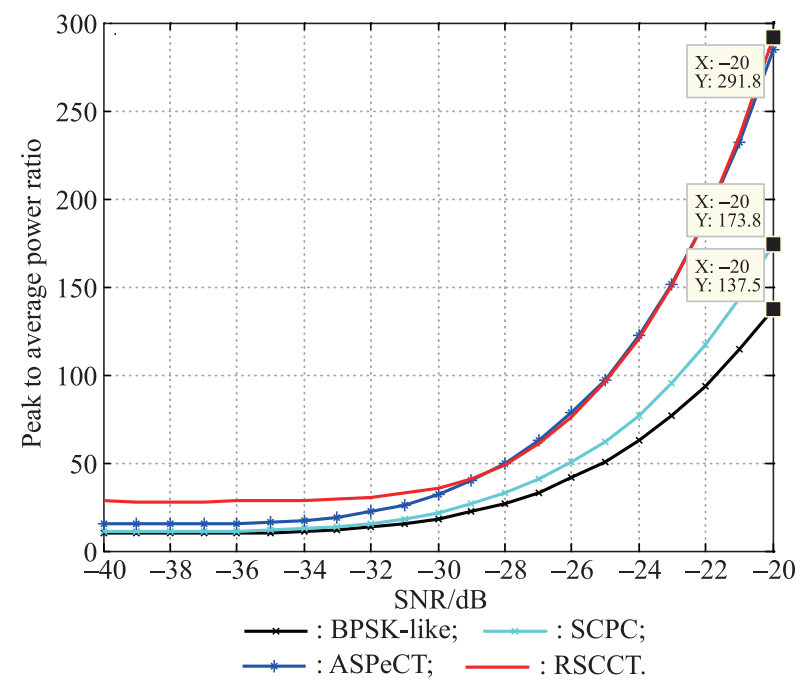

Fig. 14 Peak to average power ratio at $N=4$

\section{Conclusions}

In this paper, an unambiguous acquisition algorithm based on reconstructing the correlation function for $\mathrm{BOC}(k n, n)$ is proposed. After the PRN code is decomposed according to the order of the BOC signal, the first sub-PRN code and the $N$ th sub-PRN code are taken out as local codes to complete the unambiguous acquisition of the BOC. Theoretical analysis and simulations show that RSCCT has the advantages of BPSK-like and SCPC's versatility and ASPeCT's narrow correlation peaks. It is applicable to all $\mathrm{BOC}(k n, n)$ signals and can take advantage of $\mathrm{BOC}$ modulation with order. Although the computational complexity of RSCCT has no obvious advantage over BPSKlike, SCPC and ASPeCT, the acquisition probability and the peak to average power ratio are greatly improved. For $\operatorname{BOC}(n, n)$, the acquisition sensitivity of RSCCT is 3.25 $\mathrm{dB}, 0.81 \mathrm{~dB}$ and $0.25 \mathrm{~dB}$ higher than BPSK-like, SCPC and $\mathrm{ASPeCT}$ at the acquisition probability of $90 \%$, respectively. The peak to average power ratio is $1.91,3.0$ and 3.7 times higher than ASPeCT, SCPC and BPSK-like at $\mathrm{SNR}=-20 \mathrm{~dB}$, respectively. For $\operatorname{BOC}(2 n, n)$, the acquisition sensitivity of RSCCT is $5.5 \mathrm{~dB}, 1.25 \mathrm{~dB}$ and $2.69 \mathrm{~dB}$ higher than BPSK-like, SCPC and ASPeCT at a acquisition probability of $90 \%$, respectively. The peak to average power ratio is $1.02,1.68$ and 2.12 times higher than ASPeCT, SCPC and BPSK-like at SNR $=-20 \mathrm{~dB}$, respectively. The above data show that this method has good acquisition performance and can be extended to all $\operatorname{BOC}(k n, n)$ signals. And most modern GNSS uses evenorder BOC. Therefore, the RSCCT algorithm proposed in this paper has a certain reference value for the acquisition technology of modern GNSS receivers.

\section{References}

[1] LIU W, HU Y, ZHAN X Q. Generalised binary offset carrier modulations for global navigation satellite systems. Electronics Letters, 2012, 48(5): $284-286$.

[2] BORIO D. Coherent side-band BOC processing. IET Radar, Sonar \& Navigation, 2017, 11(10): 1455-1466.

[3] ZHU Y B, BAO L I, RUI L, et al. An improved code phase acquisition algorithm for BOC signal. Geomatics \& Information Science of Wuhan University, 2015, 40(3): 406-411. (in Chinese)

[4] LOHAN E S. Statistical analysis of BPSK-like techniques for the acquisition of galileo signals. Journal of Aerospace Computing Information \& Communication, 2012, 3(5): 234-243.

[5] ZHANG H. Research and implementation of the tracking algorithm of BOC-modulated signal. Beijing, China: University of Chinese Academy of Sciences, 2015. (in Chinese)

[6] QIAN S J, YIN X F. Research on ASPeCT-based acquisition and tracking of BOC modulating signal. Modern Electronics Technique, 2016, 39(21): 65-69.

[7] ZHANG T Q, YAN Z H, OU X D, et al. Acquisition algorithm combining parallel code phase with fractal-reconstruction of TMBOC modulation signal. Journal of Shanghai Jiao Tong University, 2016, 50(5): $782-787$.

[8] JI Y F, LIU Y, ZHEN W M, et al. An unambiguous acquisition algorithm based on unit correlation for $\operatorname{BOC}(n, n)$ signal. IEICE Trans. on Communications, 2017, E100-B(8): 1507 1513.

[9] GÓMEZ-CASCO D, GARCIA-MOLINA J A, GUSI-AMIGÓ A, et al. Mitigation of false locks in the acquisition of highorder BOC signals in HS-GNSS receivers. Proc. of the International Conference on Localization \& GNSS, 2016: 1-6.

[10] SHEN F, XU G H, FENG H Y, et al. Synthesized correlation function based unambiguous acquisition techique for sin-BOC/MBOC modulated singals. Systems Engineering and Electronics, 2015, 37(9): 1980-1986. (in Chinese)

[11] WANG L F. Research on method of signal acquisition and tracking under new modulation in GNSS. Tianjin: Civil Aviation University of China, 2015.

[12] LEI D, LU W, YU D. Resource-efficient acquisition architecture for BOC-modulated signals. IEICE Electronics Express, 2014, 11(11): 20140358 -20140358.

[13] ARIAS-CASTRO E, BUBECK S, LUGOSI G, et al. Detecting Markov random fields hidden in white noise. Bernoulli, 2015, 24(4): $3628-3656$.

[14] REN J W, YANG G T, JIA W M, et al. Unitary unambiguous tracking method based on combined correlation functions for BOC modulation signals. Acta Aeronautica et Astronautica Sinica, 2014, 35(7): 2031-2040. (in Chinese)

[15] ZHENG K, ZHANG X, UNIVERSITY B. An unambiguous acquisition algorithm for improving pseudo code phase measurement precision of BOC signal. Journal of Telemetry Tracking \& Command, 2018, 39(2): $41-47$.

[16] CAO X L, GUO C J, ZHANG X X, et al. An acquisition algorithm based on combined correlation functions for $\operatorname{BOC}(n, n)$ signals. Electronics Optics \& Control, 2017(3): 51-54. 


\section{Biographies}

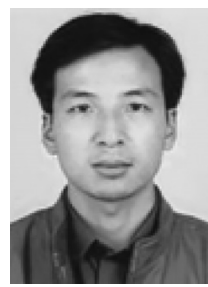

JI Yuanfa was born in 1975. He received his Ph.D. degree in Science and Engineering from National Astronomical Observatories, Chinese Academy of Sciences in 2008. He now stays in Guangxi Key Laboratory of Precision Navigation Technology and Application to do research in satellite navigation and signal processing.

E-mail: jiyuanfa@163.com

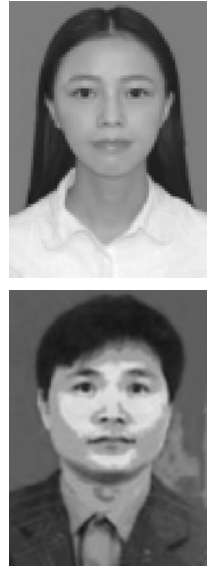

CHEN Xiaoqian was born in 1994. She is a postgraduate student in Guilin University of Electronic Technology. She now stays in Guangxi Key Laboratory of Precision Navigation Technology and Application to study satellite navigation and signal processing.

E-mail: 870743923@qq.com

FU Qiang was born in 1976. He received his M.S. degree from Guilin University of Electronic Technology in 2007. He now stays in Guangxi Key Laboratory of Precision Navigation Technology and Application to do research in satellite navigation and image processing.

E-mail: fqrfh@guet.enu.cn

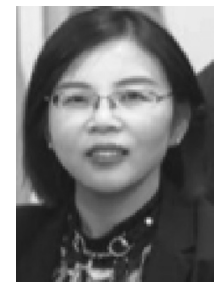

SUN Xiyan was born in 1973. She received her M.S. degree from Guilin University of Electronic Technology in 2002 and Ph.D. degree in science and engineering from National Astronomical Observatories, Chinese Academy of Sciences in 2006. From 2006 to 2014, she stayed with Guangxi Key Laboratory of Precision Navigation Technology and Application to do research in satellite navigation and signal processing. She is the author of two books, more than 100 articles, and more than 30 inventions. She is now working at the Guangxi Experiment Center of Information Science.

E-mail: sunxiyan1@163.com

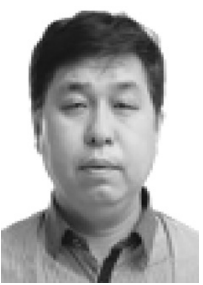

ZHEN Weimin was born in 1980. He is a researcher-level senior engineer. Now he works as an expert of the BeiDou system and serves as the chairman of the International Committee on Global Navigation Satellite Systems (ICG), and chairs the project of electromagnetic interference detection and weakening.

E-mail: Crirp_zwm@163.com 